\title{
On Borel Classes of Sets of Fréchet Subdifferentiability
}

\author{
by
}

\section{Ondřej KURKA}

Presented by Czestaw BESSAGA

Summary. We study possible Borel classes of sets of Fréchet subdifferentiability of continuous functions on reflexive spaces.

1. Introduction and main result. Our terminology follows $[2,6]$. We recall the most important definitions and notation in Sections 1 and 2. In this paper, all normed linear spaces are supposed to be real.

Let $X$ be a normed linear space and $f$ be a real function on $X$. For $x \in X$, we define the Fréchet subdifferential of $f$ at $x$ by

$$
\partial f(x)=\left\{u \in X^{*}: \liminf _{y \rightarrow x} \frac{f(y)-f(x)-u(y-x)}{\|y-x\|} \geq 0\right\} .
$$

Any element of $\partial f(x)$ is called a Fréchet subgradient of $f$ at $x$. We say that $x$ is a point of Fréchet subdifferentiability of $f$ if $\partial f(x) \neq \emptyset$. The set of all points of Fréchet subdifferentiability of $f$ is denoted by $S(f)$.

First, we recall a known result in this area.

THEOREM 1.1 (Holický, Laczkovich). Let $f$ be a lower semicontinuous function on a normed linear space $X$ with reflexive completion. Then $S(f)$ is a $\Sigma_{4}^{0}$ set.

The proof of this theorem can be found in [3]. Note that the set of Fréchet subdifferentiability of a continuous function on a normed linear space $X$ may not be Borel if the completion of $X$ is not reflexive (see [3, Theorem 1.3]).

The main result of the paper follows. It says that the result of Holický and Laczkovich is "best possible".

2000 Mathematics Subject Classification: 26B05, 54H05.

Key words and phrases: set of Fréchet subdifferentiability, $\Sigma_{4}^{0}$-complete set. 
Theorem 1.2. Let $X$ be a normed linear space with $\operatorname{dim} X \geq 3$. Then there is a continuous real function $f$ on $X$ such that $S(f)$ is $\Sigma_{4}^{0}$-complete.

This theorem will be proved in Section 3 .

REMARK 1.3. 1) If $f$ is a continuous function on $\mathbb{R}$, then $S(f)$ is a $\Pi_{3}^{0}$ set by the classical result that the Dini derivatives of $f$ are of Baire class 2 (see, e.g., [1]). On the other hand, by a result of Zahorski (see, e.g., [5]), there is a Lipschitz function $f$ on $\mathbb{R}$ such that the set $D(f)$ of all points of differentiability of $f$ is $\Pi_{3}^{0}$-complete. Since $D(f)=S(f) \cap S(-f)$, at least one of the sets $S(f), S(-f)$ is $\Pi_{3}^{0}$-complete.

If $f: \mathbb{R}^{2} \rightarrow \mathbb{R}$ is lower semicontinuous, then $S(f)$ is $\Sigma_{4}^{0}$ (by Theorem 1.1) and can be $\Pi_{3}^{0}$-complete (by the result of Zahorski). We do not know anything more about the situation in $\mathbb{R}^{2}$.

2) The set of Fréchet subdifferentiability of a Lipschitz function $f$ on a space with reflexive completion is a $\Pi_{3}^{0}$ set. This follows from the proof of Theorem 1.2 in [3] and from the observation that the norms of Fréchet subgradients of $f$ are uniformly bounded by the Lipschitz constant (and thus $S(f)=\bigcap_{k=1}^{\infty} \bigcup_{\left(n_{1}, \ldots, n_{k}\right) \in \mathbb{N}^{k}} A_{n_{1}, \ldots, n_{k}}^{K}$ for some $K \in \mathbb{N}$, where $A_{n_{1}, \ldots, n_{k}}^{K}=$ $\left.\bigcup_{\|u\| \leq K} \bigcap_{i=1}^{k}\left\{x \in X:\|y-x\|<1 / n_{i} \Rightarrow f(y)-f(x) \geq u(y-x)-i^{-1}\|y-x\|\right\}\right)$. Together with the above-mentioned result of Zahorski, this says that $\Pi_{3}^{0}$ is the smallest Borel class which contains the set of Fréchet subdifferentiability of each Lipschitz function on a reflexive space.

3) Let $g: X \rightarrow[-\infty, \infty)$ be a lower semicontinuous function, where $X$ is a space with reflexive completion. Then the set $G=\{x \in X: g(x)>-\infty\}$ is open, and $S(g) \subset G$ can be defined in the same way as $S(f)$ for finite $f$. By the method of Holický and Laczkovich, $S(g) \in \Sigma_{4}^{0}$.

4) Let $X$ be a space with reflexive completion and $f: X \rightarrow \mathbb{R}$ be $\Sigma_{\alpha^{-}}^{0}$ measurable (i.e., $f^{-1}(U) \in \Sigma_{\alpha}^{0}$ whenever $U \subset \mathbb{R}$ is open). One may ask whether $S(f)$ is Borel, or even of which Borel class it is. By an observation of Šmídek (see [4]), $S(f)=S(g) \cap\{x \in X: f(x)=g(x)\}$, where $g$ is the greatest lower semicontinuous minorant of $f$. So $S(f)$ is the intersection of a $\Sigma_{4}^{0}$ set and a $\Pi_{\alpha}^{0}$ set.

2. Some elements of descriptive set theory. Let us recall some definitions and notation. A topological space is called Polish if it is separable and completely metrizable.

Given a topological space $M$, we use $\Sigma_{\alpha}^{0}(M)$ and $\Pi_{\alpha}^{0}(M)$, where $\alpha<\omega_{1}$, for the Borel classes (see [2]). What is most important for us is that $\Sigma_{4}^{0}$ is $F_{\sigma \delta \sigma}$ and $\Pi_{3}^{0}$ is $F_{\sigma \delta}$ in the classical notation. We say that $A \subset M$ is $\Sigma_{\alpha}^{0}$-hard (resp. $\Pi_{\alpha}^{0}$-hard) if, for every zero-dimensional Polish space $P$ and $B \in \Sigma_{\alpha}^{0}(P)$ (resp. $B \in \Pi_{\alpha}^{0}(P)$ ), there exists a continuous mapping $f: P \rightarrow M$ such that 
$f^{-1}(A)=B$. We say that $A$ is $\Sigma_{\alpha}^{0}$-complete (resp. $\Pi_{\alpha}^{0}$-complete) if $A$ is $\Sigma_{\alpha}^{0}$-hard and $A \in \Sigma_{\alpha}^{0}(M)$ (resp. $\Pi_{\alpha}^{0}$-hard and $A \in \Pi_{\alpha}^{0}(M)$ ).

Let $P$ be a Polish space. It is known that being $\Sigma_{\alpha}^{0}$-complete in $P$ amounts to being an element of $\Sigma_{\alpha}^{0}(P) \backslash \Pi_{\alpha}^{0}(P)$ (and similarly for $\Sigma_{\alpha}^{0}$ and $\Pi_{\alpha}^{0}$ interchanged). For example, a subset of $\mathbb{R}^{3}$ is $\Sigma_{4}^{0}$-complete if and only if it is $F_{\sigma \delta \sigma}$, but not $G_{\delta \sigma \delta}$.

By $\forall^{\infty}$ we mean "for all but finitely many".

Lemma 2.1 (cf. [2, Exercise 23.3]). The set

$$
D=\left\{\nu \in\{0,1\}^{\mathbb{N} \times \mathbb{N} \times \mathbb{N}}: \forall^{\infty} k \in \mathbb{N} \forall m \in \mathbb{N} \forall^{\infty} l \in \mathbb{N} \nu(k, l, m)=0\right\}
$$

is $\Sigma_{4}^{0}$-hard in $\{0,1\}^{\mathbb{N} \times \mathbb{N} \times \mathbb{N}}$.

Proof. By $[2,23 . \mathrm{A}]$, the set

$$
E=\left\{\sigma \in\{0,1\}^{\mathbb{N} \times \mathbb{N}}: \forall m \in \mathbb{N} \forall^{\infty} l \in \mathbb{N} \sigma(l, m)=0\right\}
$$

is $\Pi_{3}^{0}$-complete in $\{0,1\}^{\mathbb{N} \times \mathbb{N}}$. Let $P$ be a zero-dimensional Polish space and $B \in \Sigma_{4}^{0}(P)$. Then $B=\bigcup_{k=1}^{\infty} B_{k}$ for some $B_{1}, B_{2}, \ldots \in \Pi_{3}^{0}(P)$. Since the class $\Pi_{3}^{0}$ is closed under finite unions, we may suppose that $B_{1} \subset B_{2} \subset \cdots$. For every $k \in \mathbb{N}$, there exists a continuous mapping $f_{k}: P \rightarrow\{0,1\}^{\mathbb{N} \times \mathbb{N}}$ such that $f_{k}^{-1}(E)=B_{k}$. We define

$$
f(p)(k, l, m)=f_{k}(p)(l, m), \quad p \in P, k, l, m \in \mathbb{N} .
$$

It is easy to check that $f: P \rightarrow\{0,1\}^{\mathbb{N} \times \mathbb{N} \times \mathbb{N}}$ is continuous and that $f^{-1}(D)=B$, which proves the lemma.

3. Proof of Theorem 1.2. In this section, by c-Lipschitz we mean Lipschitz with constant $c$.

Lemma 3.1. There are continuous functions $\chi_{k, l}: \mathbb{R} \rightarrow[0,1], k, l \in \mathbb{N}$, such that

(a) $\chi_{k, l}(x) \geq \chi_{k+1, l}(x)$ for every $k, l \in \mathbb{N}, x \in \mathbb{R}$,

(b) $\chi_{k, l}$ is l-Lipschitz for every $k, l \in \mathbb{N}$,

(c) the set

$$
\bigcup_{k=1}^{\infty}\left\{x \in \mathbb{R}: \lim _{l \rightarrow \infty} \chi_{k, l}(x)=0\right\}
$$

is $\Sigma_{4}^{0}$-hard in $\mathbb{R}$.

Proof. We define functions $n_{k, l}:\{0,1\}^{\mathbb{N} \times \mathbb{N} \times \mathbb{N}} \rightarrow \mathbb{N}$ and $\varphi_{k, l}, \phi_{k, l}:$ $\{0,1\}^{\mathbb{N} \times \mathbb{N} \times \mathbb{N}} \rightarrow[0,1]$ for $k, l \in \mathbb{N}$ by 


$$
\begin{aligned}
n_{k, l}(\nu) & =\min (\{m \in \mathbb{N}: \nu(k, l, m)=1\} \cup\{l\}), \\
\varphi_{k, l}(\nu) & =\frac{1}{n_{k, l}(\nu)}, \\
\phi_{k, l} & = \begin{cases}0, & k>l, \\
\sum_{j=k}^{l} 2^{-j} \varphi_{j, l}, & k \leq l .\end{cases}
\end{aligned}
$$

For $k \in \mathbb{N}$ and $\nu \in\{0,1\}^{\mathbb{N} \times \mathbb{N} \times \mathbb{N}}$, we verify the following two equivalences:

$\lim _{l \rightarrow \infty} \varphi_{k, l}(\nu)=0 \Leftrightarrow \lim _{l \rightarrow \infty} n_{k, l}(\nu)=\infty \Leftrightarrow \forall m \in \mathbb{N} \forall^{\infty} l \in \mathbb{N}: \nu(k, l, m)=0$.

The first equivalence is obvious; let us prove the other one. Assume that $\lim _{l \rightarrow \infty} n_{k, l}(\nu)=\infty$. For given $m \in \mathbb{N}$, we have to find $p \in \mathbb{N}$ such that $\nu(k, l, m)=0$ for every $l \geq p$. We choose $p \in \mathbb{N}$ such that $n_{k, l}(\nu)>m$ for every $l \geq p$. By the definition of $n_{k, l}, \nu(k, l, j)=0$ for $1 \leq j \leq m$ and $l \geq p$, which gives the implication " $\Rightarrow$ ". Now, suppose

$$
\forall m \in \mathbb{N} \forall^{\infty} l \in \mathbb{N}: \nu(k, l, m)=0 .
$$

For given $m$, we have to find $p \in \mathbb{N}$ such that $n_{k, l}(\nu)>m$ for every $l \geq p$. If $l>m$, then by the definition of $n_{k, l}$ we have $n_{k, l}(\nu)>m$ whenever $\nu(k, l, i)=0$ for $1 \leq i \leq m$. So it is enough to choose $p \in \mathbb{N}$ such that $p>m$ and $\nu(k, l, i)=0$ for $l \geq p$ and for $1 \leq i \leq m$. This proves the implication " $\Leftarrow$ ", and the second equivalence is also proved.

Now, we are going to prove that

$$
\bigcup_{k=1}^{\infty}\left\{\nu \in\{0,1\}^{\mathbb{N} \times \mathbb{N} \times \mathbb{N}}: \lim _{l \rightarrow \infty} \phi_{k, l}(\nu)=0\right\}=D
$$

for the set $D$ from Lemma 2.1. Indeed, for $\nu \in\{0,1\}^{\mathbb{N} \times \mathbb{N} \times \mathbb{N}}$, we have

$$
\begin{aligned}
\nu \in D & \Leftrightarrow \exists k_{0} \in \mathbb{N} \forall k \geq k_{0} \forall m \in \mathbb{N} \forall^{\infty} l \in \mathbb{N}: \nu(k, l, m)=0 \\
& \Leftrightarrow \exists k_{0} \in \mathbb{N} \forall k \geq k_{0}: \lim _{l \rightarrow \infty} \varphi_{k, l}(\nu)=0 \\
& \Leftrightarrow \exists k_{0} \in \mathbb{N}: \lim _{l \rightarrow \infty} \sum_{j=k_{0}}^{\infty} 2^{-j} \varphi_{j, l}(\nu)=0 \\
& \Leftrightarrow \exists k_{0} \in \mathbb{N}: \lim _{l \rightarrow \infty} \phi_{k_{0}, l}(\nu)=0 .
\end{aligned}
$$

Let $\pi: \mathbb{N} \times \mathbb{N} \times \mathbb{N} \rightarrow \mathbb{N}$ be a bijection. We define a homeomorphism $h$ between $\{0,1\}^{\mathbb{N}}$ and $\{0,1\}^{\mathbb{N} \times \mathbb{N} \times \mathbb{N}}$ by

$$
h:\left(\alpha_{n}\right)_{n \in \mathbb{N}} \in\{0,1\}^{\mathbb{N}} \mapsto\left(\alpha_{\pi(k, l, m)}\right)_{(k, l, m) \in \mathbb{N} \times \mathbb{N} \times \mathbb{N}} .
$$

Consider the following metric on $\{0,1\}^{\mathbb{N}}$ :

$$
\varrho\left(\nu, \nu^{\prime}\right)=\max \left(\left\{3^{-n}: \nu(n) \neq \nu^{\prime}(n)\right\} \cup\{0\}\right), \quad \nu, \nu^{\prime} \in\{0,1\}^{\mathbb{N}} .
$$


Let us check that

(1) $\forall l \in \mathbb{N} \exists L_{l}>0 \forall k \in \mathbb{N}: \phi_{k, l} \circ h$ is $L_{l}$-Lipschitz on $\left(\{0,1\}^{\mathbb{N}}, \varrho\right)$.

It is enough to prove that there exists $L_{k, l}>0$ such that $\varphi_{k, l} \circ h$ is $L_{k, l^{-}}$ Lipschitz for every $k, l \in \mathbb{N}$ because then we can take $L_{l}=\sum_{j=1}^{l} 2^{-j} L_{j, l}$. We put

$$
L_{k, l}=\max \left\{3^{\pi(k, l, m)}: 1 \leq m \leq l\right\}, \quad k, l \in \mathbb{N} .
$$

Let $\nu, \nu^{\prime} \in\{0,1\}^{\mathbb{N}}$. If $\varrho\left(\nu, \nu^{\prime}\right) \geq 1 / L_{k, l}$, then $\left|\left(\varphi_{k, l} \circ h\right)(\nu)-\left(\varphi_{k, l} \circ h\right)\left(\nu^{\prime}\right)\right| \leq$ $1 \leq L_{k, l} \varrho\left(\nu, \nu^{\prime}\right)$. If $\varrho\left(\nu, \nu^{\prime}\right)<1 / L_{k, l}$ (i.e., $\varrho\left(\nu, \nu^{\prime}\right)<3^{-\pi(k, l, m)}$ for $1 \leq$ $m \leq l)$, then, by the definition of $\varrho, \nu(\pi(k, l, m))=\nu^{\prime}(\pi(k, l, m))$ (i.e., $\left.h(\nu)(k, l, m)=h\left(\nu^{\prime}\right)(k, l, m)\right)$ for $1 \leq m \leq l$, and, by the definitions of $n_{k, l}$ and $\varphi_{k, l}, n_{k, l}(h(\nu))=n_{k, l}\left(h\left(\nu^{\prime}\right)\right)$ and $\varphi_{k, l}(h(\nu))=\varphi_{k, l}\left(h\left(\nu^{\prime}\right)\right)$. So the choice of $L_{k, l}$ works, and (1) is proved.

Now, define $g:\{0,1\}^{\mathbb{N}} \rightarrow \mathbb{R}$ by

$$
g(\nu)=2 \sum_{k=1}^{\infty}\left(\frac{1}{3}\right)^{k} \nu(k), \quad \nu \in\{0,1\}^{\mathbb{N}} .
$$

One can easily check that

$$
\frac{1}{3}\left|g(\nu)-g\left(\nu^{\prime}\right)\right| \leq \varrho\left(\nu, \nu^{\prime}\right) \leq\left|g(\nu)-g\left(\nu^{\prime}\right)\right|, \quad \nu, \nu^{\prime} \in\{0,1\}^{\mathbb{N}} .
$$

Set $C=g\left(\{0,1\}^{\mathbb{N}}\right)$. We see that $g$ is a homeomorphism of $\{0,1\}^{\mathbb{N}}$ onto $C$. Consequently, the set

$$
\bigcup_{k=1}^{\infty}\left\{x \in C: \lim _{l \rightarrow \infty}\left(\phi_{k, l} \circ h \circ g^{-1}\right)(x)=0\right\}=g\left(h^{-1}(D)\right)
$$

is $\Sigma_{4}^{0}$-hard in $C$ by Lemma 2.1 .

Since $g^{-1}$ is 1-Lipschitz, the function $\phi_{k, l} \circ h \circ g^{-1}$ is $L_{l}$-Lipschitz for $k, l \in \mathbb{N}$. We can extend these functions from $C$ to $\mathbb{R}$ by

$\chi_{k, l}^{\prime}=\sup \left\{u: \mathbb{R} \rightarrow[0,1]: u\right.$ is $L_{l}$-Lipschitz, $u \leq \phi_{k, l} \circ h \circ g^{-1}$ on $\left.C\right\}$.

We now prove that the following conditions hold:

$\left(\mathrm{a}^{\prime}\right) \chi_{k, l}^{\prime}(x) \geq \chi_{k+1, l}^{\prime}(x)$ for every $k, l \in \mathbb{N}, x \in \mathbb{R}$,

$\left(\mathrm{b}^{\prime}\right) \chi_{k, l}^{\prime}$ is $L_{l}$-Lipschitz for every $k, l \in \mathbb{N}$,

$\left(c^{\prime}\right)$ the set

$$
\bigcup_{k=1}^{\infty}\left\{x \in \mathbb{R}: \lim _{l \rightarrow \infty} \chi_{k, l}^{\prime}(x)=0\right\}
$$

is $\Sigma_{4}^{0}$-hard in $\mathbb{R}$.

Let $k, l \in \mathbb{N}$. Obviously, $\phi_{k, l} \geq \phi_{k+1, l}$ on $\{0,1\}^{\mathbb{N} \times \mathbb{N} \times \mathbb{N}}$. Thus, $\phi_{k, l} \circ h \circ g^{-1}$ $\geq \phi_{k+1, l} \circ h \circ g^{-1}$ on $C$. Hence $\chi_{k, l}^{\prime} \geq \chi_{k+1, l}^{\prime}$ by the definitions of $\chi_{k, l}^{\prime}$ and 
$\chi_{k+1, l}^{\prime}$. So $\left(\mathrm{a}^{\prime}\right)$ holds. Since the supremum of any non-empty system of $c$ Lipschitz functions with a uniform upper bound at one point is $c$-Lipschitz, $\left(\mathrm{b}^{\prime}\right)$ holds. Finally, since $g\left(h^{-1}(D)\right)=C \cap \bigcup_{k=1}^{\infty}\left\{x \in \mathbb{R}: \lim _{l \rightarrow \infty} \chi_{k, l}^{\prime}(x)=0\right\}$ is $\Sigma_{4}^{0}$-hard in $C,\left(\mathrm{c}^{\prime}\right)$ holds.

We choose an increasing sequence of natural numbers $1 \leq s_{1}<s_{2}<\cdots$ such that $s_{i} \geq L_{i}$ for $i \in \mathbb{N}$. For $k, l \in \mathbb{N}$, we define

$$
\chi_{k, l}= \begin{cases}1, & 1 \leq l<s_{1}, \\ \chi_{k, i}^{\prime}, & s_{i} \leq l<s_{i+1}, i \in \mathbb{N}\end{cases}
$$

which completes the proof.

Proof of Theorem 1.2. It is enough to construct a function $f$ with the required properties on $\mathbb{R}^{3}$ (in the general case, $X$ can be expressed as the topological sum $\mathbb{R}^{3} \oplus Y$ for some closed subspace $Y$ of $X$, and if we define $F(x+y)=f(x)$ for $x \in \mathbb{R}^{3}$ and $y \in Y$, then $S(F)=S(f)+Y$ would also be $\Sigma_{4}^{0}$-complete). In the proof, we use $|\cdot|$ for the Euclidean norm on $\mathbb{R}^{n}, n=2,3$.

Let $\chi_{k, l}: \mathbb{R} \rightarrow[0,1], k, l \in \mathbb{N}$, be as in Lemma 3.1. We define functions $f, f_{l}: \mathbb{R}^{3} \rightarrow \mathbb{R}, l \in \mathbb{N}$, by

$$
\begin{aligned}
& f_{l}(x, y, z)=\max \left\{(k-1) y-\chi_{k, l}(x)|(y, z)|: 1 \leq k \leq l\right\}, \quad(x, y, z) \in \mathbb{R}^{3}, \\
& f(x, y, z)=\left\{\begin{array}{cc}
0, \quad y=z=0, \\
\frac{(l+1)^{-2}-|(y, z)|}{(l+1)^{-2}-(l+2)^{-2}} f_{l+1}(x, y, z) \\
+\frac{|(y, z)|-(l+2)^{-2}}{(l+1)^{-2}-(l+2)^{-2}} f_{l}(x, y, z), \\
f_{1}(x, y, z), & (l+2)^{-2} \leq|(y, z)|<(l+1)^{-2}, \\
& 1 / 4 \leq|(y, z)| .
\end{array}\right.
\end{aligned}
$$

Obviously, the functions $f_{l}, l \in \mathbb{N}$, are continuous and the function $f$ is continuous on $\left\{(x, y, z) \in \mathbb{R}^{3}:(l+2)^{-2} \leq|(y, z)|<(l+1)^{-2}\right\}, l \in \mathbb{N}$, and on $\left\{(x, y, z) \in \mathbb{R}^{3}: 1 / 4 \leq|(y, z)|\right\}$. To prove that $f$ is continuous on the union of these sets (i.e., on $\left\{(x, y, z) \in \mathbb{R}^{3}:|(y, z)|>0\right\}$ ), we have to check that for $l \in \mathbb{N}$ and $\left(x_{0}, y_{0}, z_{0}\right) \in \mathbb{R}^{3}$ with $\left|\left(y_{0}, z_{0}\right)\right|=(l+1)^{-2}$,

$$
\lim _{\substack{(x, y, z) \rightarrow\left(x_{0}, y_{0}, z_{0}\right) \\(l+2)^{-2} \leq|(y, z)|<(l+1)^{-2}}} f(x, y, z)=f\left(x_{0}, y_{0}, z_{0}\right) .
$$

This holds because both sides of the equality are equal to $f_{l}\left(x_{0}, y_{0}, z_{0}\right)$. The proof of the continuity of $f$ will be completed if we verify that

$$
|f(x, y, z)| \leq \sqrt{|(y, z)|} \quad \text { for }(x, y, z) \in \mathbb{R}^{3} \text { with }|(y, z)|<1 / 4
$$

(and thus that $f$ is continuous at each $(x, 0,0)$ for $x \in \mathbb{R})$. Let $(x, y, z) \in \mathbb{R}^{3}$ and $|(y, z)|<1 / 4$. We may suppose that $|(y, z)|>0$. Let $l \in \mathbb{N}$ be such that 
$(l+2)^{-2} \leq|(y, z)|<(l+1)^{-2}$. Since $f(x, y, z)$ is a convex combination of $f_{l}(x, y, z)$ and $f_{l+1}(x, y, z)$, it is enough to check that

$$
|(y, z)| \leq j^{-2} \Rightarrow\left|f_{j}(x, y, z)\right| \leq \sqrt{|(y, z)|}
$$

for $j \in \mathbb{N}$ (and thus $\left|f_{l}(x, y, z)\right| \leq \sqrt{|(y, z)|}$ and $\left|f_{l+1}(x, y, z)\right| \leq \sqrt{|(y, z)|}$ ). Let $j \in \mathbb{N}$ be such that $|(y, z)| \leq j^{-2}$. Using the definition of $f_{j}$ (and the fact that the ranges of $\chi_{k, j}$ are subsets of $\left.[0,1]\right)$, we get $\left|f_{j}(x, y, z)\right| \leq j|(y, z)|$. We have

$$
\left|f_{j}(x, y, z)\right| \leq j|(y, z)| \leq|(y, z)|^{-1 / 2}|(y, z)|=\sqrt{|(y, z)|},
$$

and the continuity of $f$ is proved.

Let us proceed to the investigation of $S(f)$. By Theorem 1.1, $S(f)$ is $\Sigma_{4}^{0}$. By the property (c) of the system $\left\{\chi_{k, l}\right\}_{k, l \in \mathbb{N}}$, to prove that $S(f)$ is $\Sigma_{4}^{0}$-complete, it is sufficient to prove that, for $a \in \mathbb{R}$,

$$
(a, 0,0) \in S(f) \Leftrightarrow \exists k \in \mathbb{N}: \lim _{l \rightarrow \infty} \chi_{k, l}(a)=0 .
$$

Let us prove the implication " $\Rightarrow$ ". Suppose $\lim \sup _{l \rightarrow \infty} \chi_{k, l}(a)>0$ for every $k \in \mathbb{N}$ and let $u \in\left(\mathbb{R}^{3}\right)^{*}$. We have to check that $u$ is not a Fréchet subgradient of $f$ at $(a, 0,0)$. Suppose the opposite, i.e., $u \in \partial f(a, 0,0)$. Let $\lambda \in \mathbb{R}$. By the definition of $f_{l}, l \in \mathbb{N}$, we have $f_{l}(a, 0, \lambda) \leq 0$. Consequently, $f(a, 0, \lambda) \leq 0$. We have

$$
0 \leq \liminf _{\lambda \rightarrow 0} \frac{f(a, 0, \lambda)-u(0,0, \lambda)}{|(0,0, \lambda)|} \leq \liminf _{\lambda \rightarrow 0} \frac{-u(0,0, \lambda)}{|\lambda|}=-|u(0,0,1)| .
$$

So $u(0,0,1)=0$ and

$$
u(0, y, z)=c y, \quad y, z \in \mathbb{R},
$$

where $c=u(0,1,0)$. We choose $n \in \mathbb{N}$ such that $n \geq c+1$. There exists $\varepsilon>0$ such that $(c+1) \varepsilon<\lim \sup _{l \rightarrow \infty} \chi_{n, l}(a)$. If we define

$$
p_{l}=(l+1)^{-2}\left(0,-\varepsilon, \sqrt{1-\varepsilon^{2}}\right), \quad l \in \mathbb{N},
$$

and use the property (a), we have

$$
\begin{aligned}
& \frac{f\left((a, 0,0)+p_{l}\right)-u\left(p_{l}\right)}{\left|p_{l}\right|}=\frac{f_{l}\left((a, 0,0)+p_{l}\right)-u\left(p_{l}\right)}{\left|p_{l}\right|} \\
& \quad=\frac{1}{(l+1)^{-2}}\left(\max \left\{(1-k)(l+1)^{-2} \varepsilon-\chi_{k, l}(a)(l+1)^{-2}: k \leq l\right\}\right)+c \varepsilon \\
& \quad \leq \sup \left\{(1-k) \varepsilon-\chi_{k, l}(a): k \in \mathbb{N}\right\}+c \varepsilon \\
& \leq \max \left\{\max \left\{(1-k) \varepsilon-\chi_{k, l}(a): 1 \leq k \leq n\right\}+c \varepsilon,-n \varepsilon+c \varepsilon\right\} \\
& \quad \leq \max \left\{c \varepsilon-\chi_{n, l}(a),-\varepsilon\right\} .
\end{aligned}
$$


By the choice of $\varepsilon$, for every $l_{0} \in \mathbb{N}$, there exists $l \geq l_{0}$ such that $\chi_{n, l}(a) \geq$ $(c+1) \varepsilon$, i.e., $c \varepsilon-\chi_{n, l}(a) \leq-\varepsilon$. Consequently,

$$
\frac{1}{\left|p_{l}\right|}\left(f\left((a, 0,0)+p_{l}\right)-u\left(p_{l}\right)\right) \leq-\varepsilon
$$

for such $l$. Since $p_{l} \rightarrow(0,0,0)$,

$$
\liminf _{(x, y, z) \rightarrow(a, 0,0)} \frac{1}{|(x-a, y, z)|}(f(x, y, z)-u(x-a, y, z)) \leq-\varepsilon
$$

which contradicts the fact that $u$ is a Fréchet subgradient of $f$ at $(a, 0,0)$. So the implication " $\Rightarrow$ " is proved.

Now, let us prove " $\Leftarrow$ ". We have to find a Fréchet subgradient of $f$ at $(a, 0,0)$ assuming that there exists $k \in \mathbb{N}$ such that $\lim _{l \rightarrow \infty} \chi_{k, l}(a)=0$. Let us fix such a $k$. We claim that

$$
u(x, y, z)=(k-1) y, \quad(x, y, z) \in \mathbb{R}^{3},
$$

is the required Fréchet subgradient. Let $\varepsilon>0$ be given. We can choose $l_{0} \in \mathbb{N}$ such that $\chi_{k, l}(a) \leq \varepsilon / 2$ for every $l \geq l_{0}$. We choose $\delta>0$ such that

$$
\delta<1 / 4, \quad \delta^{1 / 2} \leq \varepsilon, \quad \delta^{1 / 6} \leq \varepsilon / 2, \quad \delta<\left(l_{0}+1\right)^{-2}, \quad \delta<(k+1)^{-2} .
$$

Let $(x, y, z) \in \mathbb{R}^{3}$ and $0<|(x-a, y, z)| \leq \delta$. We now check that

$$
\frac{f(x, y, z)-u(x-a, y, z)}{|(x-a, y, z)|} \geq-\varepsilon .
$$

Clearly, this holds if $(y, z)=0$. So we may suppose that $|(y, z)|>0$. For $l \geq k$, by the definition of $f_{l}$,

$$
f_{l}(x, y, z)-(k-1) y \geq-\chi_{k, l}(x)|(y, z)| .
$$

Since $0<|(y, z)| \leq \delta<1 / 4$, we have $(l+2)^{-2} \leq|(y, z)|<(l+1)^{-2}$ for some $l \in \mathbb{N}$. Since $(l+2)^{-2} \leq|(y, z)| \leq \delta<(k+1)^{-2}$, it follows that $l \geq k$. Since $f(x, y, z)$ is a convex combination of $f_{l}(x, y, z)$ and $f_{l+1}(x, y, z)$, it follows that

$$
f(x, y, z)-u(x-a, y, z) \geq-\max \left\{\chi_{k, l}(x)|(y, z)|, \chi_{k, l+1}(x)|(y, z)|\right\} .
$$

If $|(y, z)| \leq|x-a|^{3 / 2}$, using (2), we have

$$
\frac{f(x, y, z)-u(x-a, y, z)}{|(x-a, y, z)|} \geq-\frac{|(y, z)|}{|(x-a, y, z)|} \geq-|x-a|^{1 / 2} \geq-\delta^{1 / 2} \geq-\varepsilon .
$$

In the other case (i.e., if $|(y, z)|>|x-a|^{3 / 2}$ ), by (b) and by the fact that $l \geq l_{0}\left((l+2)^{-2} \leq|(y, z)| \leq \delta<\left(l_{0}+1\right)^{-2}\right)$, using (2) again, we have

$$
\begin{aligned}
\frac{f(x, y, z)-u(x-a, y, z)}{|(x-a, y, z)|} & \geq-\frac{\max \left\{\chi_{k, l}(x)|(y, z)|, \chi_{k, l+1}(x)|(y, z)|\right\}}{|(x-a, y, z)|} \\
& \geq-\max \left\{\chi_{k, l}(x), \chi_{k, l+1}(x)\right\}
\end{aligned}
$$




$$
\begin{aligned}
& \geq-\max \left\{\chi_{k, l}(a), \chi_{k, l+1}(a)\right\}-(l+1)|x-a| \\
& >-\varepsilon / 2-|(y, z)|^{-1 / 2}|(y, z)|^{2 / 3} \\
& \geq-\varepsilon / 2-\delta^{1 / 6} \geq-\varepsilon .
\end{aligned}
$$

So, for given $\varepsilon>0$, we have found $\delta>0$ such that

$$
0<|(x-a, y, z)| \leq \delta \Rightarrow \frac{f(x, y, z)-u(x-a, y, z)}{|(x-a, y, z)|} \geq-\varepsilon .
$$

This means that $u$ is a Fréchet subgradient of $f$ at $(a, 0,0)$, and the implication " $\Leftarrow$ " is proved.

I would like to thank Petr Holický for helpful discussions and useful remarks.

\section{References}

[1] A. M. Bruckner, Differentiation of Real Functions, Lecture Notes in Math. 659, Springer, 1978.

[2] A. S. Kechris, Classical Descriptive Set Theory, Grad. Texts in Math. 156, Springer, New York, 1995.

[3] O. Kurka, Reflexivity and sets of Fréchet subdifferentiability, 2007/230 at http:// www.karlin.mff.cuni.cz/kma-preprints/.

[4] M. Šmídek, Measurability of some subsets of spaces of functions, Charles Univ., 1994 (in Czech).

[5] Z. Zahorski, Sur la première dérivée, Trans. Amer. Math. Soc. 69 (1950), 1-54.

[6] L. Zajíček, Fréchet differentiability, strict differentiability and subdifferentiability, Czechoslovak Math. J. 41 (1991), 471-489.

Ondřej Kurka

Department of Mathematical Analysis

Faculty of Mathematics and Physics

Charles University

Sokolovská 83

18675 Praha 8, Czech Republic

E-mail: ondrej.kurka@mff.cuni.cz

Received June 17, 2007;

received in final form September 3, 2007 